\title{
Musidunin and Musiduol, Insect Antifeedants from Croton jatrophoides
}

Ken-ichi Nihei, ${ }^{\dagger}$ Yukihiro Asaka, ${ }^{\ddagger}$ Yoshihiro Mine,${ }^{\S}$ Yoichi Yamada, ${ }^{\perp}$ Masayuki ligo, ${ }^{\dagger}$ Tadashi Yanagisawa, $^{\dagger}$ and Isao Kubo $\|, *$

Department of Applied Biochemistry, Faculty of Agriculture, Utsunomiya University, Utsunomiya, Tochigi 321-0943, Japan, Department of Life Sciences, Faculty of Science and Technology, Kinki University, Higashi-osaka, Osaka 577-8502, Japan, Life Science Research Institute, Kinki University School of Medicine, Sayama, Osaka 589-8511, Japan, Department of Chemistry, Faculty of Education, Utsunomiya University, Utsunomiya, Tochigi 321-0943, Japan, and Department of Environmental Science, Policy and Management, University of California, Berkeley, California 94720-31122,

\section{Supporting Information}

Figure S1. Significant COLOC correlations for 1.

Figure S2. Significant nOe correlations for 1 . The dashed and solid lines express nOe correlations on $\alpha$-face and $\beta$-face, respectively.

Figure S3. Selected nOe and COLOC correlations for 2. The dashed line with double-headed arrow expresses nOe correlation. The single-headed arrow represents COLOC correlation. Figure S4. Possible biogenetic pathway of 1 from dumsenin. 


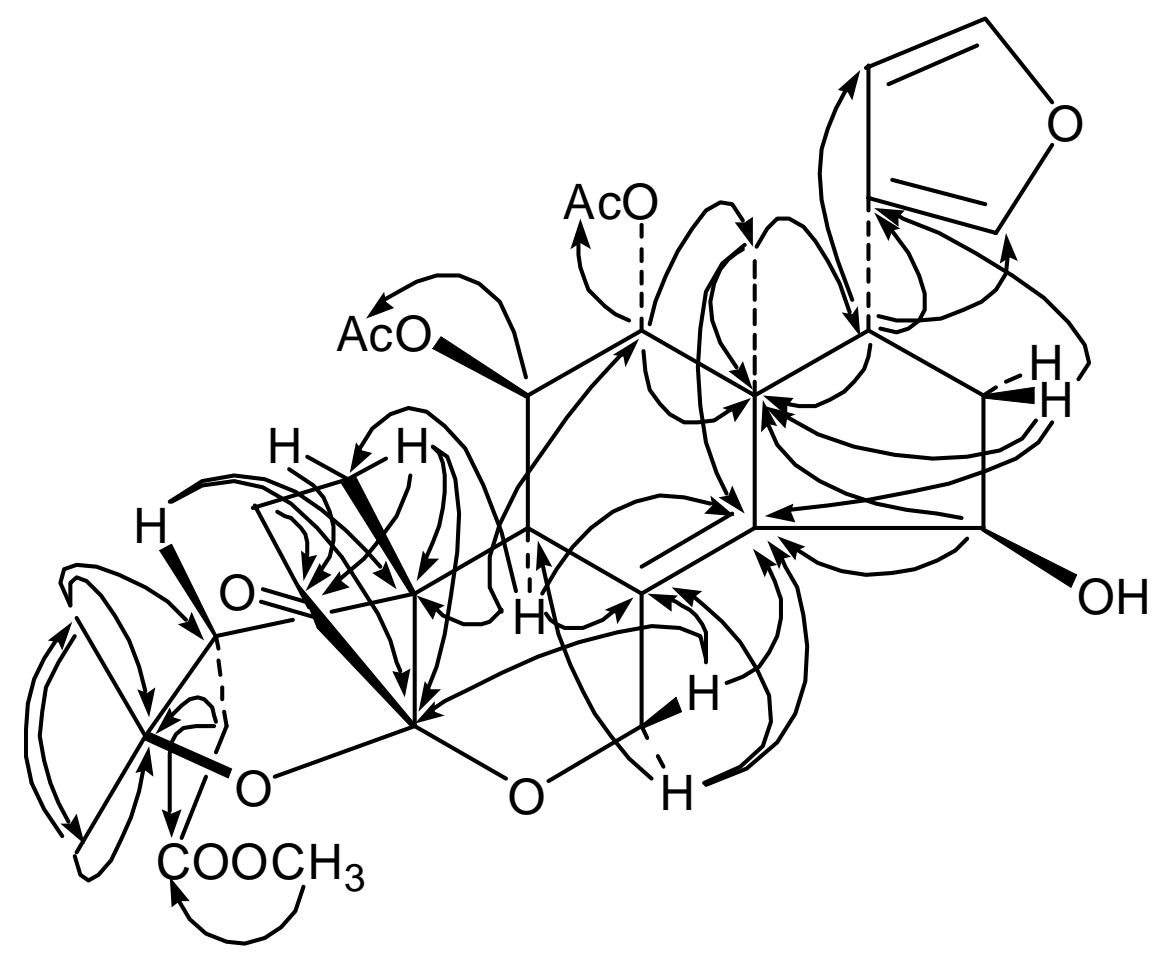

Figure S1. Significant COLOC correlations for $\mathbf{1 .}$ 


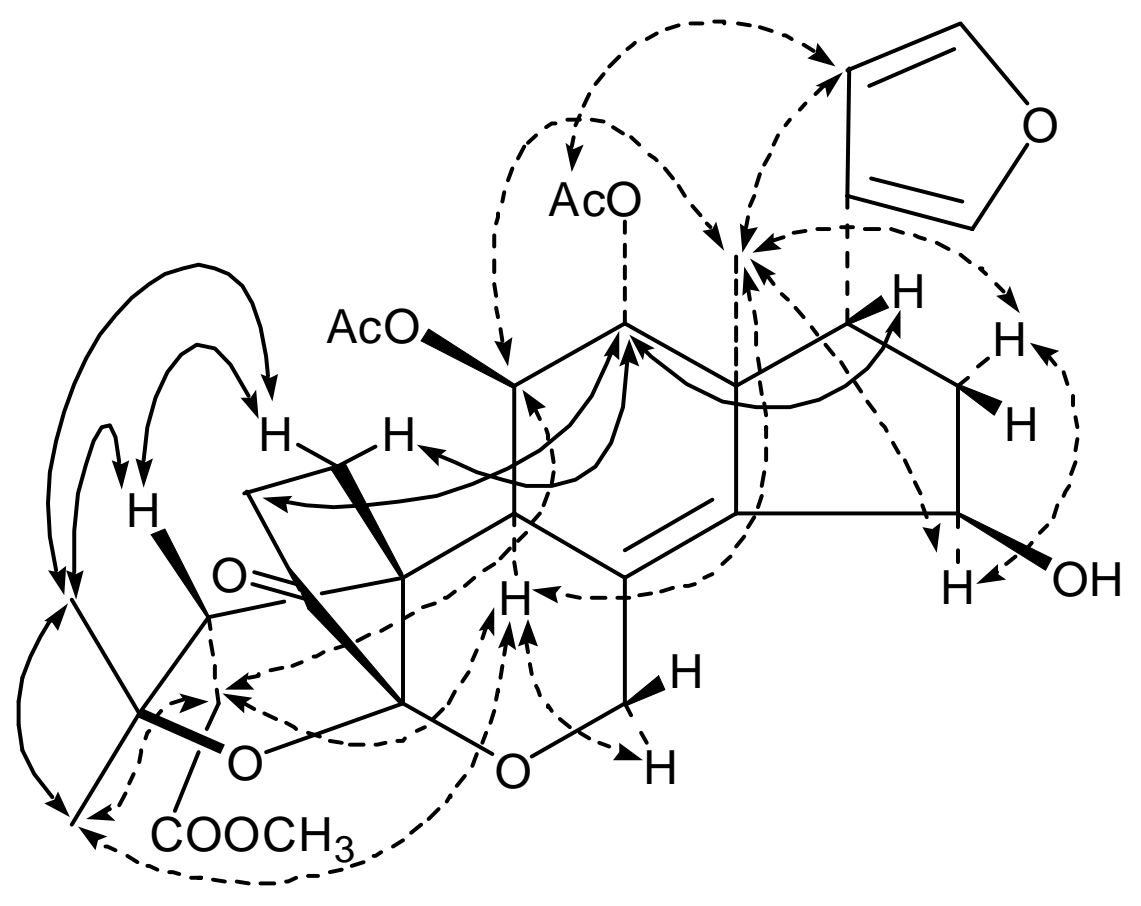

Figure S2. Significant nOe correlations for $\mathbf{1}$. The dashed and solid lines express nOe correlations on $\alpha$-face and $\beta$-face, respectively. 


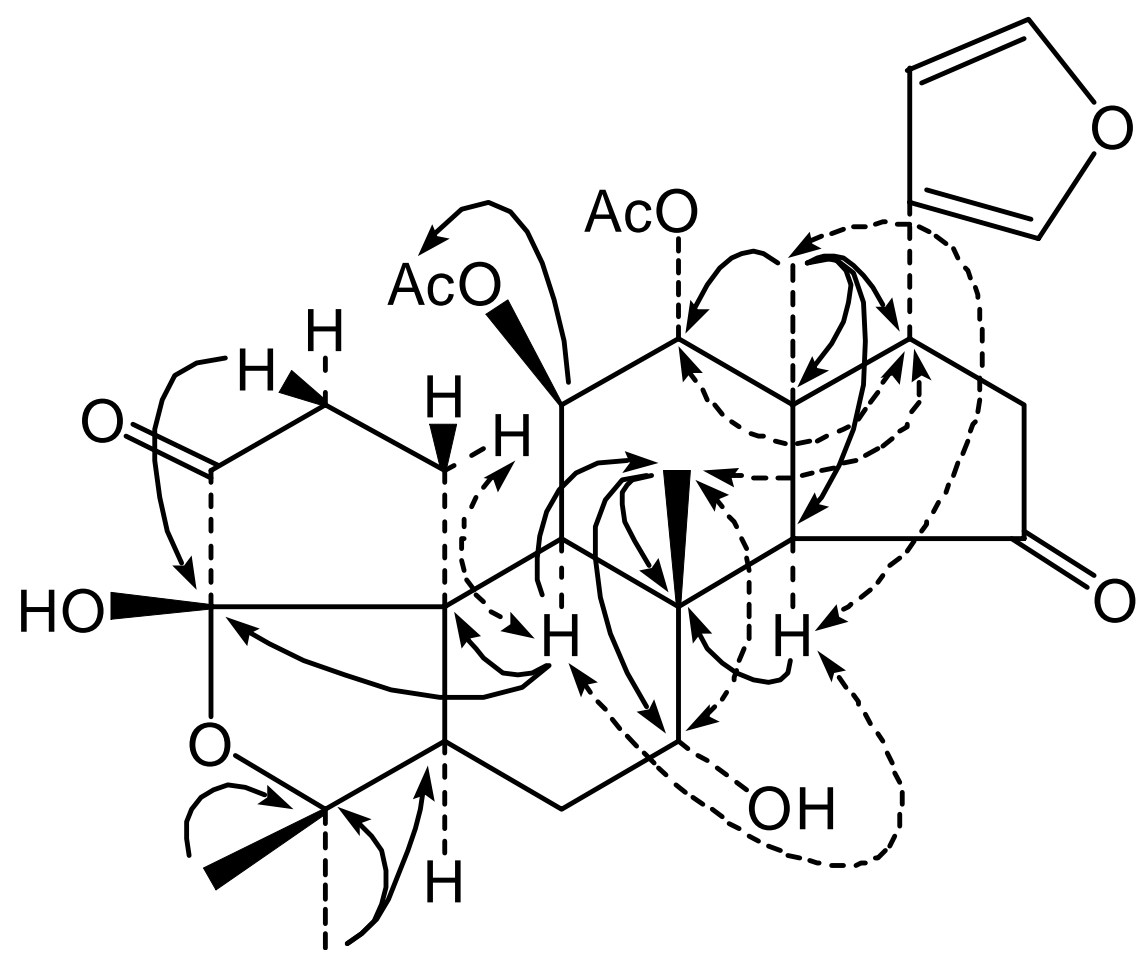

Figure S3. Selected nOe and COLOC correlations for $\mathbf{2}$. The dashed line with double-headed arrow expresses nOe correlation. The single-headed arrow represents COLOC correlation. 

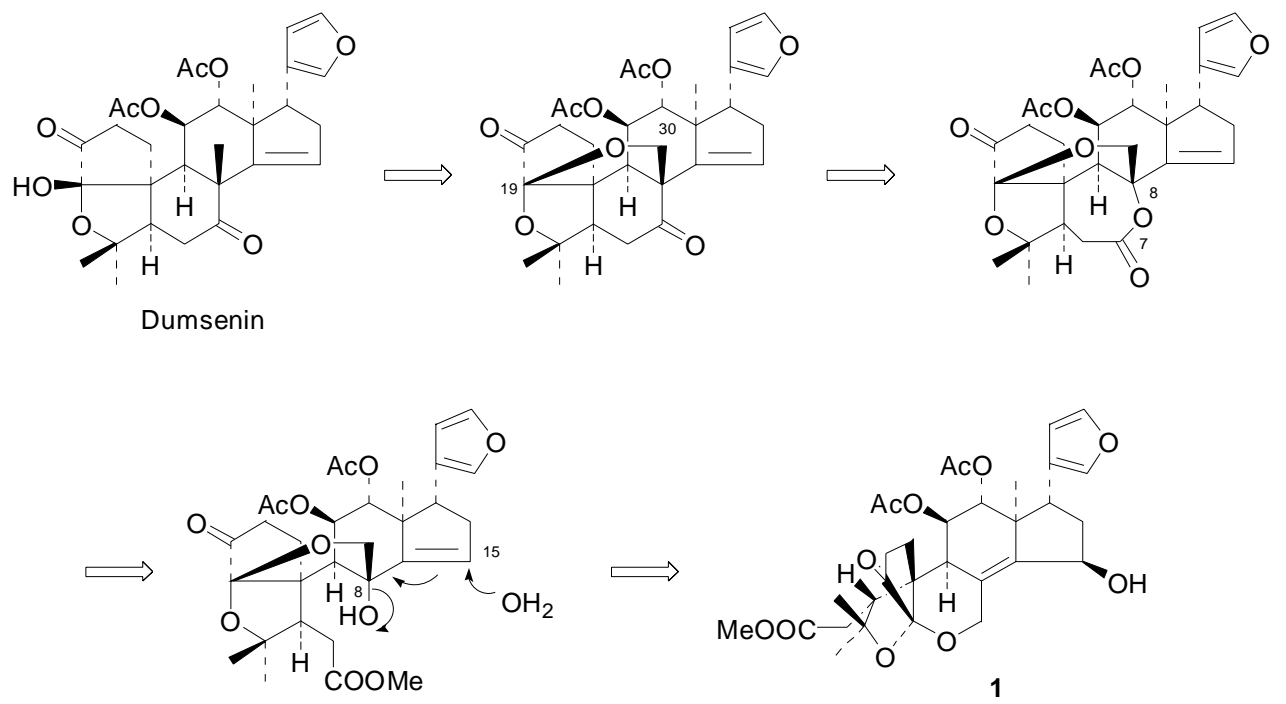

Figure S4. Possible biogenetic pathway of $\mathbf{1}$ from dumsenin. 